\title{
Design of a New 8k x 8k Lens Coupled Detector for Wide-field, High-resolution Transmission Electron Microscopy.
}

Steven T. Peltier, James C. Bouwer, Liang Jin, Krist Khodjasaryan, Steve Geist, NguyenHuu Xuong, and Mark H. Ellisman.

National Center for Microscopy and Imaging Research, Center for Research in Biological Systems, University of California at San Diego, La Jolla, CA 92093-0608

In this presentation, we will present the details underlying the design, development, and fabrication of a large-format high-resolution digital imaging detector for transmission electron microscopy (TEM). This system incorporates 4-high performance demagnification lenses to split the image from a self-supported scintillator screen onto

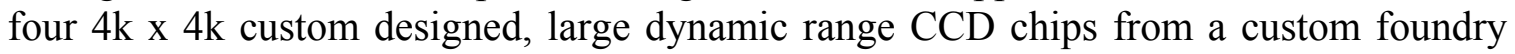
run at Fairchild Imaging. The format of this detector is approximately $7.5 \mathrm{k} \times 7.5 \mathrm{k}$ depending on sub-image overlap. The ultimate goal of this project is to replace film as the resolution standard for TEM.

Building on our previous experience with lens-coupled digital camera systems, we have produced a design that moves the lens-coupled design to the next logical step (Fan and Ellisman, 2000; Fan et al., 1996, Fan et al. 1994, Downing et al., 1999). (Figure 1) The readout characteristics of this system are that of a 16 parallel port device reading out at nearly $1 \mathrm{Mhz} /$ port, producing 64 Megapixels in four seconds. The CCD cameras are being produced by Spectral Imaging in Tucson AZ. By using 1.5:1 demagnification lenses to match the scintillator point spread function to the pixel pitch of the CCD cameras we achieve the proper 1:1 correspondence between an electron spot and a CCD pixel. An alternative to this technology is to use a tapered fiber to map the large electron footprint on the scintillator to the pixel pitch of the CCD. However, tapered fibers introduce distortions and point dislocations into the image that make them unsuitable for high resolution imaging systems (Faruqi et al. 1999). Coupling this with large numerical aperture lenses built by Optics1 in Westlake CA and a sizable modular transfer amplitude at the Nyquist frequency of the CCD, we expect to produce a final contrast at Nyquist of about $15-20 \%$, while still retaining single electron sensitivity.

While a tremendous effort was made to produce the full system specification, an equal effort was made to engineer the system mechanical design to meet that specification and to accommodate the mechanical constraints of the TEM itself. In particular, a new superstructure and support apparatus was developed to physically support the device within the frame of a modern IVEM while achieving the drop distance necessary to provide proper scintillator coverage. A large scintillator is necessary to provide image overlap for the final image stitching. Custom flanging is central to this design to seat the scintillator correctly and interface the optics of the device to the vacuum of the microscope.

The mechanical alignment and adjustment of the system optics is also critical to providing proper performance. Custom low profile stages have been built to provide lens alignment including lens translation, pitch, yaw, and focusing without appreciable increasing the height of the bottom-mount system. A separate coupled stage system is used to provide 5 axis CCD camera alignments. The entire system is designed to be 
highly modular and repeatable. Each element of the system has been designed for ease of removal for maintenance, adjustment, and/or upgrade. Scintillators can also be easily swapped in and out to customize the system for high sensitivity or high resolution depending on the application. Custom lens shutter cartridges have also been installed to provide easy removal and reinstallation.

With the large amount of data generated by the system, a custom computer control and acquisition system was required and has been developed. In particular, high performance image capture boards have been used to reassemble the sub-images and present the final image. The system includes software to interact with the microscope to provide beam blanking, tilting, image translation, and focusing.

A critical challenge to the engineering design of any detector system for high voltage applications is x-ray safety. Shielding of system components has been carefully considered and preliminary components will be presented.

Downing, KH; Hendrickson, FM. (1999) Performance of a 2K CCD camera designed for electron crystallography at $400 \mathrm{kV}$, Ultramicroscopy 75: 215-33.

Fan G.Y. et al. \& M.H. Ellisman (2000) Multiport-read-out frame-transfer 5 megapixel CCD imaging system for TEM applications, Ultramicroscopy, submitted.

Fan, G.Y. and M. Ellisman (1996) Optimization of thin foil based phosphor screens for CCD imaging in TEM in the voltage range of 80 to $400 \mathrm{KeV}$, Ultramicroscopy 66, 11-19.

Fan, G. Y., Dana G. Dunkelberger, and Mark Ellisman (1994) Performance of a thin foil scintillating screen for transmission electron microscopy, Ultramicroscopy 55, 7-14.

Faruqi, A.R., R. Henderson, S. Subramaniam (1999) Cooled CCD detector with tapered fiber optics for recording electron diffraction patterns, Ultramicroscopy 75, 235-250.

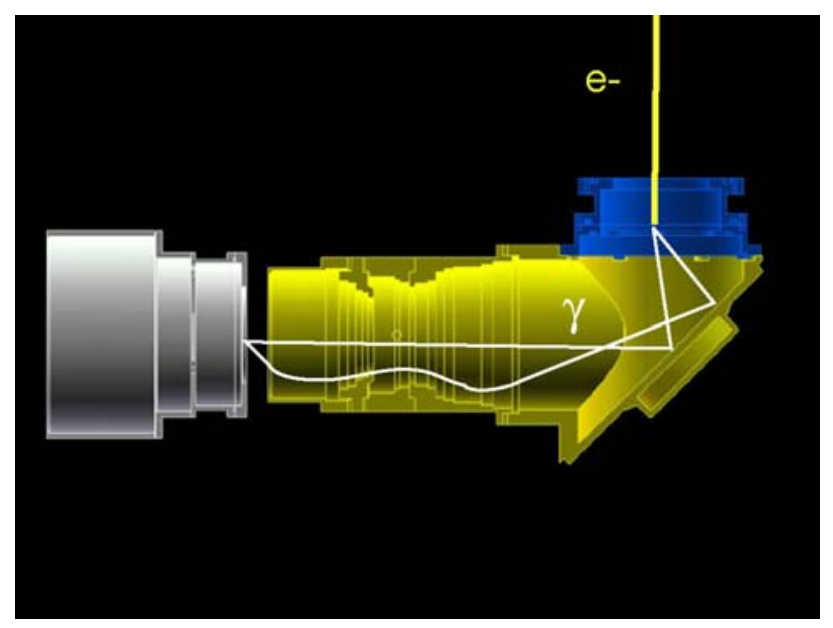

Figure 1. Our original $2 \mathrm{k}$ x $2.5 \mathrm{k}$ high-resolution lens coupled camera design. This design was the highest resolution, largest format system at the time it was build. It still remains the highest resolution system at $400 \mathrm{keV}$, although it lacks the large format of more modern systems. 